\title{
Niveles de boro no permisibles en reservorio de agua potable, cuenca hidrográfica Locumba, Región Tacna-Perú
}

Levels of boron not permissible in reservoir of drinking water, Locumba hydrographic basin, Tacna-Peru Region

Recibido: junio 11 de 2017 | Revisado: julio 20 de 2017 | Aceptado: setiembre 23 de 2017

Dante U. Morales Cabrera ${ }^{\mathrm{I}}$ Edgardo Avendaño Cáceres ${ }^{I}$ Daniel Zevallos Ramos ${ }^{\mathrm{I}}$ Julio Fernández Prado ${ }^{\mathrm{I}}$ Zoila L. Mendoza Rodas ${ }^{1}$ Amparo Torres Ventura ${ }^{\mathrm{I}}$
1 Universidad Nacional Jorge Basadre Grohmann. Región Tacna, Perú moralesdante@hotmail.com

\section{RESUMEN}

La toxicidad del boro sigue siendo de conocimiento limitado, aunque estudios experimentales indican efectos en el sistema reproductivo de manera que, el conocimiento sobre sus concentraciones en agua potable es de prioridad para la regulación ambiental. El propósito de este estudio fue determinar los niveles de boro en reservorio de agua potable de la cuenca hidrográfica Locumba, Región Tacna-Perú. Se realizó un muestreo probabilístico aleatorio en los meses de agosto y noviembre del 2016, encontrándose concentraciones de boro $\left(8,681\right.$ y $\left.4,148 \mathrm{mg} . \mathrm{L}^{-1}\right)$ por encima al valor regulado $\left(2,4 \mathrm{mg} \cdot \mathrm{L}^{-1}\right)$. La prueba t-Student indicó diferencias estadísticamente significativas $(\mathrm{p} \geq 0.05)$ entre los valores determinados. Se concluyó que, las concentraciones de boro en el reservorio de agua potable durante los meses de agosto y noviembre son un riesgo para la salud humana.

Palabras clave: boro, toxicidad, agua potable, cuenca hidrográfica locumba, Tacna-Perú

\section{Abstract}

The toxicity of boron is still of limited knowledge, although experimental studies indicate its effects on the reproductive system so that the knowledge about its concentrations in drinking water is a priority and needed for environmental regulation. The purpose of this study was to determine the levels of boron in the drinking water reservoir of the Locumba basin, Tacna-Peru Region. A random probabilistic sampling was performed in August and November 2016, with boron concentrations (8.681 and 4,148 mg.L $\mathrm{L}^{-1}$ ) above the regulated value $\left(2.4 \mathrm{mg} \cdot \mathrm{L}^{-1}\right)$. The $\mathrm{t}$-Students test indicated statistically significant differences $(p \geq 0.05)$ between the determined values. It was concluded that the concentrations of boron in the drinking water reservoir during August and November were undesirable, which could represent a risk t human health when consumed.

Key words: boron, toxicity, drinking water, basin hydrographic basin, Tacna-Peru 


\section{Introducción}

El boro es un microelemento esencial para las plantas (Yilmaz, Boncukcuoğlu, Kocakerim, Yilmaz \& Paluluoğlu, 2008) como para los animales (Dydo, Turek, Ciba, Trojanowska \& Kluczka, 2005) en la actualidad, sigue siendo un tema de interés y debate debido a su efecto perjudicial (Mustafa \& Sirri, 2013). A pesar que las concentraciones permisibles varían de un país a otro (Hilal, Kim \& Somerfield, 2011), la Organización Mundial de la Salud (2011), refiere que entre 0,1$0,3 \mathrm{mg} \cdot \mathrm{L}^{-1}$ oscilan las concentraciones en agua potable, asignando un valor de referencia provisional de $0,5 \mathrm{mg} \cdot \mathrm{L}^{-1}$, así como 2,4 mg. $\mathrm{L}^{-1}$ para el consumo humano (Tagliabue, Reverberi \& Bagatin, 2014).

En las aguas naturales destinadas a su potabilización, la composición química es variable y ello obedece a la geología (Benko \& Drewes, 2008). Por lo general, el boro siempre está presente en las aguas potables (Ezechi, Isa \& Mohamed, 2012), lo que se debe a que es utilizado comúnmente como reticulante ya que la concentración del agua de retorno puede ser mayor que el agua de formación (Barati \& Liang, 2014). La remoción del boro es un campo novedoso con mayor énfasis a el uso de tecnología (Dydo \& Turek, 2013; Güler, Kaya, Kabay \& Arda, 2015). La eliminación del exceso de boro es un proceso costoso y requiere de mucha eficiencia de manera que, la determinación temprana contribuye no solo al tipo de tratamiento sino además, a identificar su uso potencial (Floquet, Sieben, MacKay \& Mostowfi, 2015).

El propósito de este estudio fue determinar los niveles de boro en reservorios de agua potable de la cuenca hidrográfica de Locumba, Región Tacna-Perú.

\section{Materiales y métodos}

Entre los meses de agosto y noviembre del 2016 se realizó un muestreo probabilístico aleatorio en dos puntos de exposición, correspondientes a la estación de muestreo: reservorio agua dulce, ITE (Tabla 1).

Tabla 1

Coordenadas georreferenciales de los puntos de muestreo

\begin{tabular}{cccc}
\hline $\begin{array}{c}\text { Estación de } \\
\text { monitoreo }\end{array}$ & Norte & Este & Altitud \\
\hline punto - I & 8024163 & 291851 & 174 \\
punto - II & 8024268 & 291865 & 191 \\
\hline
\end{tabular}

La determinación de boro fue mediante el método SM4500 B "Standar method for the examintion of water and waste water APHA-AWWA, 2012, que se realizó en el Laboratorio Analítico JYR S.A.C, especializado en el área ambiental, acreditado bajo la Norma NTP ISO/IEC 17025 ante la Dirección de Acreditación

del Instituto Nacional de Calidad (INACAL-DA).

Para el tratamiento de los resultados, se aplicó como método estadístico el análisis t-Student, a fin de examinar las diferencias entre dos muestras independientes que presentaron distribución normal y homogeneidad en sus varianzas según lo expresado por Fagerland (2012) y Sánchez (2015). Todos los cálculos se realizaron utilizando el software Statgraphics (Stapoint Technologies, 1994-2001), donde los resultados se consideraron significativos a un nivel de confianza del $95 \%(\mathrm{p} \leq 0.05)$. 


\section{Resultados}

La Tabla 2 muestra los resultados de boro en agosto y noviembre comparados con la referencia ambiental donde las determinaciones analíticas superaron el valor permisible, recomendado por el De- creto Supremo No. 015-2015-MINAM, Estándares Nacionales de Calidad Ambiental para Agua. República del Perú (Categoría 1: aguas superficiales destinadas a la producción de agua potable; subcategoría 2: aguas que pueden ser potabilizadas con tratamiento convencional).

Tabla 2

Valores de boro / meses

\begin{tabular}{ccc}
\cline { 2 - 3 } & agosto & noviembre \\
\hline Referencia $\left(2,4 \mathrm{mg} . \mathrm{L}^{-1}\right)$ & 8,681 & 4,148 \\
\hline
\end{tabular}

En la Tabla 3, se muestra el resumen estadístico y la comparación entre las determinaciones de boro. Como el sesgo estandarizado y la curtosis estandarizada se encontraron en el rango establecido $(-2 a+2)$ existieron desviaciones significativas dentro de la normalidad y dado que al comparar las medias mediante la Prueba t, el intervalo no contenía el valor 0 , existió una diferencia estadísticamente significativa entre las medias de boro para los dos meses con un nivel de confianza del $95.0 \%$.

Tabla 3

\section{Resumen estadistico}

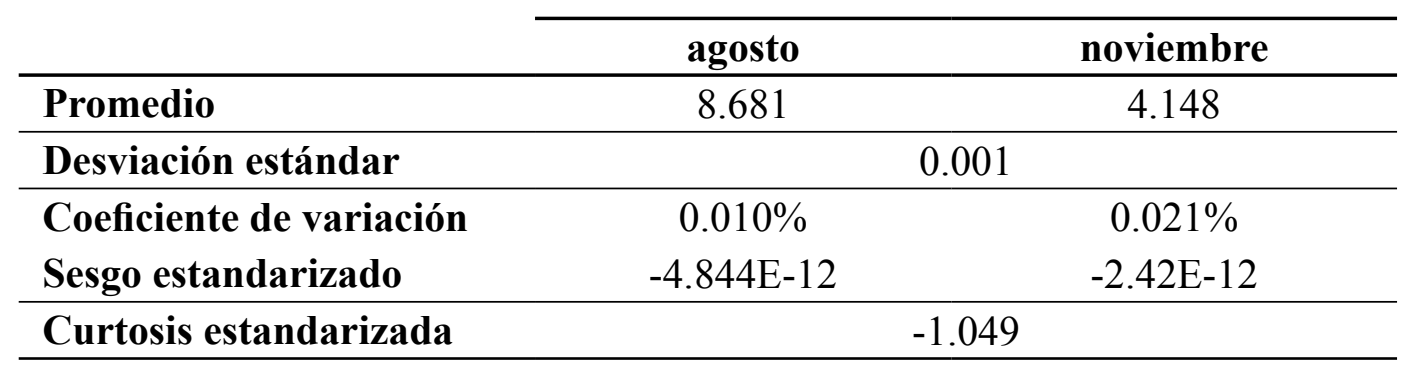

\section{Comparación de medias}

Intervalos de confianza del $95.0 \%$ intervalo de confianza para la diferencia de medias, suponiendo varianzas iguales: $4.533+/-0.001$ [4.532, 4.533]

\section{Prueba t para comparar medias}

suponiendo varianzas iguales: $\mathrm{t}=11103.5$, valor- $\mathrm{P}=0.0$ 


\section{Discusión}

Las determinaciones de boro, en este estudio, superaron el valor permisible por la norma ambiental utilizada de manera que, existe un riesgo para su valor de uso. A pesar de que, el boro puede ser tratado, en agua potable, utilizando técnicas convencionales como procesos de membranas, coagulación, electrocoagulación, adsorción e intercambio iónico (Hilal, Kim \& Somerfield, 2011), no existen reportes sobre ninguna de estas tecnologías para eliminar la contaminación por boro en las aguas superficiales de las principales cuencas hidrográficas en la Región Tacna. Wolska \& Bryjak (2013) señalan que las tecnologías convencionales mencionadas pueden remover hasta el $90 \%$ del boro en aguas residuales, aunque del mismo modo, logran que se garanticen los estándares de calidad que exige cualquier normativa. Hasenmueller y Criss (2013), Türker Türe, Böcük y Yakar (2016) y Türker Türe, Böcük, Cicek y Yakar (2016) refieren que cuando las concentraciones de boro en agua potable son bajas, las técnicas convencionales, además, de ser costosas e insostenibles, producen residuos secundarios durante la purificación. Teniendo en cuenta que las concentraciones encontradas fueron elevadas, alguna de estas técnicas puede implementarse debido, al riesgo humano por exposición y consumo permanente que existe.

Aunque no se determinó la forma química del boro en el agua potable, algunos estudios in-vitro han demostrado que el ácido bórico, ácido fenilborónico y fructoborato de calcio señalan hacia propiedades proliferativas de las líneas celulares tanto para cáncer de mama y próstata (Bradke, Hall, Carper \& Plopper, 2008; Scorei et al., 2008). Estudios toxicológicos experimentales han aludido que el boro tiene efectos negativos sobre la reproducción masculina, al encontrarse bajo número y tamaño de los espermatozoides (Robinns et al., 2010; Igra, Harari, Lu, Casimiro \& Vahter, 2016). Por su parte, Niu, Kasparov y Strongina (1972) informaron que a dosis bajas, la mayor sensibilidad está en el sistema reproductor masculino y donde finalmente; Robinns et al. (2008) mencionan que a exposiciones altas de boro, la mayor progenie no se daría para los hombres, ya que el cromosoma $Y$, es el más afectado.

\section{Conclusiones}

Las concentraciones de boro, en el reservorio de agua potable durante agosto y noviembre no son deseadas por lo que representan un riesgo por consumo a la salud humana.

\section{Agradecimientos}

A la Universidad Nacional Jorge Basadre Grohmann (UNJBG), Región de Tacna-Perú por el financiamiento con recursos del canon minero a la investigación sobre el proyecto: Análisis y modulación de tecnologías de aguas modernas para la remoción de arsénico y boro en las cuencas de la región de Tacna; así como, al Dr. George Argota Pérez, Director General del Centro de Investigaciones Avanzadas y Formación Superior en Educación, Salud y Medio Ambiente "AMTAWI", por su asesoramiento metodológico. 


\section{Referencias}

Benko, K.L. \& Drewes, J.E. (2008). Produced water in the Western United States: Geographical distribution, occurrence, and composition. Environmental Engineering Science, 25, 239-246. doi: https://doi. org/10.1089/ees.2007.0026

Barati, R. \& Liang, J.T. (2014). A review of fracturing fluid systems used for hydraulic fracturing of oil and gas Wells. Journal of Applied Polymer Science, 131. doi: https://dx.doi. org/10.1002/app.40735

Bradke, T.M., Hall, C., Carper, S.W. \& Plopper, G.E. (2008). Phenylboronic acid selectively inhibits human prostate and breast cancer cell migration and decreases viability. Cell Adhesion \& Migration, 2(3), 153-160. Recuperado de https:// www.ncbi.nlm.nih.gov/pmc/articles/PMC2634091/

Decreto Supremo No. 015-2015MINAM. Estándares Nacionales de Calidad Ambiental para Agua. Ministerio del Ambiente, República del Perú. Recuperado en http:// www.ana.gob.pe/sites/default/files/ normatividad/files/ds-ndeg-0152015-minam.pdf

Dydo, P. \& Turek, M. (2013). Boron transport and removal using ion-exchange membranes: A critical review. Desalination; 310, 2-8. doi:https://doi.org/10.1016/j.desal.2012.08.024
Dydo, P., Turek, M., Ciba, J., Trojanowska, J. \& Kluczka, J. (2005). Boron removal from landfill leachate by means of nanofiltration and reverse osmosis. Desalination, 185, 131-137. doi:http://10.1016/j.desal.2005.03.076

Ezechi, E.H., Isa, M.H. \& Mohamed, K.S.R.B. (2012). Boron in produced water: Challenges and improvements: A comprehensive review. Journal Applied Sciences; 12, 402-415. Disponible en: http:// docsdrive.com/pdfs/ansinet/ jas/2012/402-415.pdf

Fagerland, M.W. (2012). T-tests, non-parametric tests, and large studies--a paradox of statistical practice? BMC Medical Research Methodology; 12, 78. doi:https:// doi.org/10.1186/1471-2288-1278

Floquet, F.A.C., Sieben, J.V., MacKay, A.B. \& Mostowfi, F. (2015). Determination of boron in produced water using the carminic acid assay. Talanta, 150, 240-252. doi:http://dx.doi.org/10.1016/j.talanta.2015.12.010

Güler, E., Kaya, C., Kabay, N. \& Arda, M. (2015). Boron removal from seawater: State-of-theart review. Desalination, 356, 85-93. doi: http://10.1016/j.desal.2014.10.009 
Hasenmueller, E.A. \& Criss, R.E. (2013). Multiple sources of boron in urban surface waters and groundwaters. Science of the total environment, 447, 235-247. doi:https://doi.org/10.1016/j.scitotenv.2013.01.001

Hilal, N., Kim, G.J. \& Somerfield, C. (2011). Boron removal from saline water: A comprehensive review. Desalination, 273, 23-35. doi:http://10.1016/j.desal.2014.10.009

Igra, A.M., Harari, F., Lu, Y., Casimiro, E. \& Vahter, M. (2016). Boron exposure through drinking water during pregnancy and birth size. Environment International, 95, 54-60. doi: http://10.1016/j.envint.2016.07.017

Mustafa, K. \& Sirri, A.M. (2013). Biological effects of boron. Reviews of environmental contamination and toxicology. USA: Springer New York.

Niu, T., Kasparov, A.A. \& Strongina, O.M. (1972). Effect of boric acid on the sexual function in males. Gig Tr Prof Zabol, 16(11), 13-16.

Robbins, A.W., Wei, F., Elashoff, A.D., Wu, GU, Xun, L. \& Jia, J. (2008). Y:X Sperm Ratio in Boron-Exposed Men. Journal of Andrology, 29(1), 115-121. Recuperado de http://onlinelibrary.wiley.com/doi/10.2164/ jandrol.107.003541/pdf

Robbins, W.A., Xun, L., Jia, J., Kennedy, N., Elashoff, D.A. \& Ping, L. (2010). Chronic boron exposure and human semen parameters. Reproductive Toxicology, 29, 184190. doi: http://10.1016/j.reprotox.2009.11.003

Sánchez, T.R.A. (2015). t-Student. Usos y abusos. Revista Méxicana de Cardiologia; 26(1), 59-61. Recuperado de http://www.medigraphic.com/ pdfs/cardio/h-2015/h151i.pdf

Scorei, R., Ciubar, R., Ciofrangeanu, C.M., Mitran, V., Cimpean, A. \& Iordachescu D. (2008). Comparative effects of boric acid and calcium fructoborate on breast cancer cells. Biol Trace Elem Res, 122(3), 197-205. doi: http://10.1007/ s12011-007-8081-8

Statgraphics Plus for Windows: SGPW. (2001). Version 5.1. Copyright 1994-2001 for Statistical Graphics Corporation

Tagliabue, M., Reverberi, A.P. \& Bagatin, R. (2014). Boron removal from water: Needs, challenges and perspectives. Journal Cleaner Production, 77, 56-64. doi:http://10.1016/j. jclepro.2013.11.040

Türker, O.C., Türe, C., Böcük, H. \& Yakar, A. (2016). Phyto-management of boron mine effluent using native macrophytes in mono-culture and poly-culture constructed wetlands. Ecological Engineering, 94, 65-74. doi: http://10.1016/j. ecoleng.2016.05.043

Türker, O.C., Türe, C., Böcük, H., Çiçek, A. \& Yakar, A. (2016). Role of plants and vegetation 
structure on boron (B) removal process in constructed wetlands. Ecological Engineering, 88, 143152. doi: http://10.1016/j.ecoleng.2015.12.021

WHO. World Health Organization. (2011). Guidelines for Drinking Water Quality, First Addendum To Third Edition. Recuperado de http://apps.who.int/iris/bitstream/ 10665/44584/1/9789241548151_ eng.pdf
Wolska, J. \& Bryjak, M. (2013). Methods for boron removal from aqueous solutions-A review. Desalination, 310, 18-24. doi:http://10.1016/j. desal.2012.08.003

Yilmaz, A.E., Boncukcuoğlu, R., Kocakerim, M.M., Yilmaz, M.T. \& Paluluoğlu, C. (2008). Boron removal from geothermal waters by electrocoagulation. J. Hazard. Mater., 153, 146-151. doi:http://10.1016/j. jhazmat.2007.08.030 